\title{
SEMICLASSICAL ASYMPTOTICS OF EIGENVALUES FOR NON-SELFADJOINT OPERATORS AND QUANTIZATION CONDITIONS ON RIEMANN SURFACES
}

\author{
Anna I. Esina ${ }^{b}$, Andrei I. Shafarevich ${ }^{a *}$ \\ ${ }^{a}$ M.V. Lomonosov Moscow State University, Leninskie Gory, 1, Moscow, Russia \\ ${ }^{b}$ Institute for Problems in Mechanics, Russian Academy of Sciences, Prospekt Vernadskogo, 101, Moscow, Russia \\ * corresponding author: shafarev@yahoo.com
}

\begin{abstract}
This paper reports a study of the semiclassical asymptotic behavior of the eigenvalues of some nonself-adjoint operators that are important for applications. These operators are the Schrödinger operator with complex periodic potential and the operator of induction. It turns out that the asymptotics of the spectrum can be calculated using the quantization conditions. These can be represented as the condition that the integrals of a holomorphic form over the cycles on the corresponding complex Lagrangian manifold, which is a Riemann surface of constant energy, are integers. In contrast to the real case (the Bohr-Sommerfeld-Maslov formulas), in order to calculate a chosen spectral series, it is sufficient to assume that the integral over only one of the cycles takes integer values, and different cycles determine different parts of the spectrum.
\end{abstract}

KEYWORDS: semiclassical asymptotics, quantization conditions, Riemann surface, spectral graph.

\section{INTRODUCTION}

One of the main problems of the semiclassical theory (see, for example, [1]) is the description of the asymptotic behavior of the spectrum of operators of the form $\hat{H}=H\left(x,-\imath h \frac{\partial}{\partial x}\right), h \rightarrow 0$. In this case, the problem can naturally be divided into two subproblems, namely:

(1.) to solve the spectral equation approximately, i.e., to find numbers $\lambda$ and functions $\psi$, satisfying the following equation for some $N>1$ :

$$
\widehat{H} \psi=\lambda \psi+O\left(h^{N}\right) ;
$$

(2.) to choose numbers of the form $\lambda$ that approach spectral points of the operator $\hat{H}$, i.e., to choose points $\lambda$ such that

$$
\left|\lambda-\lambda_{0}\right|=O\left(h^{N}\right)
$$

for some point $\lambda_{0}$ of the spectrum of operator $\hat{H}$.

If operator $\hat{H}$ is self-adjoint, then the estimate (2) automatically follows from equation (1) (see, e.g., [13]). At the same time, the first problem is highly nontrivial and is related to the study of invariant sets of the corresponding classical Hamiltonian system. Recall how to solve this problem (1) in the integrable case. Let

$$
H(x, p): \mathbb{R}^{2 n} \rightarrow \mathbb{R}
$$

be a smooth function, and let the Hamiltonian system defined by function $H$ be Liouville integrable. Let $f_{1}=H, \ldots, f_{n}$ be the commuting first integrals; consider the domain of the phase space smoothly fibered into Liouville tori $\Lambda$ which are the compact connected components of the common level sets of the form $f_{j}=c_{j}$. We assume that the Weyl operator $\hat{H}$ is self-adjoint in $L^{2}\left(\mathbb{R}_{x}^{n}\right)$. The following theorem is due to V. P. Maslov.

Theorem 1. Suppose that a Liouville torus $\Lambda$ satisfies the following conditions (the so-called BohrSommerfeld-Maslov quantization rules, see [1, 2, 4, 5]):

$$
\frac{1}{2 \pi h} \int_{\gamma}(p, d x)=m+\frac{\mu(\gamma)}{4}
$$

where $m=O(1 / h) \in \mathbb{Z}, \gamma$ is an arbitrary cycle on $\Lambda$, and $\mu(\gamma)$ is the Maslov index of the cycle. Then there is a function $\psi \in L^{2}\left(\mathbb{R}^{n}\right),\|\psi\|=1$, such that

$$
\hat{H} \psi=\lambda \psi+O\left(h^{2}\right), \quad \lambda=\left.H\right|_{\Lambda} .
$$

Remark 1 . The function $\psi$ mentioned in the theorem can be described in a computable way, namely, it is of the form $K(1)$, where $K$ stands for the Maslov canonical operator on the Liouville torus $\Lambda$. Integer $m$ can be chosen in the form $m=[1 / h]_{\text {int }}+m_{0}$, where $[1 / h]_{\text {int }}$ stands for the integral part of the real number $1 / h$ and $m_{0}$ does not depend on $h$.

Remark 2. As was already noted above, it follows automatically from the statement of the theorem that the point $\lambda$ is at a distance of the order of $O\left(h^{2}\right)$ from the spectrum of operator $\hat{H}$.

Remark 3. We stress that the topological condition (1.3) must be satisfied for all cycles of torus $\Lambda$ (in other words, the quantization condition is the condition that the cohomology class

$$
\frac{1}{2 \pi h}[\theta]-\frac{1}{4}[\mu]
$$

is integer, where $[\theta]$ stands for the class of the form $(p, d x)$ and $[\mu]$ for the Maslov class). 
Remark 4. In action-angle variables $\left(I_{1}, \ldots\right.$, $\left.I_{n}, \varphi_{1}, \ldots, \varphi_{n}\right)$, the quantization conditions and formula for the spectrum have a simple form (see e.g. [2])

$$
I_{j}=h\left(m_{j}+\frac{\mu_{j}}{4}\right), \quad \lambda=H\left(I_{1}, \ldots, I_{n}\right) .
$$

The nonself-adjoint case has been investigated less, and quite incompletely; however, spectral problems for nonself-adjoint operators arise in many important physical applications (like the theory of hydrodynamic stability, a description of magnetic fields of the Earth and of galaxies, $\mathcal{P} \mathcal{T}$-symmetric quantum theory, statistical mechanics of Coulomb gases, and many other problems; see, for example, 6 [11]).

In our paper, we consider two classes of nonselfadjoint operators, namely, the one-dimensional Schrödinger operator with complex potential and the operator of magnetic induction on a two-dimensional symmetric surface. The spectrum of these operators, in the semiclassical limit, is concentrated in the $O\left(h^{2}\right)$ neighborhood of some curves in the complex plane $E$; these curves form the so-called spectral graph. It turns out that each edge of the spectral graph corresponds to a certain cycle on the Riemann surface defined by the classical complex Hamiltonian system (this is a surface of constant energy).

The asymptotics of the eigenvalues can be calculated by using complex equations which are similar to the Bohr-Sommerfeld-Maslov quantization conditions on the Riemann surface. However, in contrast to the self-adjoint case, in order to evaluate the eigenvalues, it is required to satisfy the corresponding condition on only one cycle, and it turns out that different cycles determine different parts of the spectrum (and different edges of the spectral graph).

\section{SCHRÖDINGER EQUATION WITH A COMPLEX POTENTIAL}

The spectral problem for the Schrödinger equation on a circle with a purely imaginary potential

$$
-h^{2} \psi^{\prime \prime}+\imath V(x) \psi=\lambda \psi, \quad \psi(x+2 \pi)=\psi(x)
$$

arises, in particular, as a model problem for the OrrSommerfeld operator in the theory of hydrodynamic stability (see, e.g., [12 20]). A close problem appears in the statistical mechanics of the Coulomb gas (see [11]). Here $h \rightarrow 0$ is a small parameter and $V(x)$ is a trigonometric polynomial. The asymptotic behavior of the spectrum of this operator for different trigonometric polynomials $V$ as $h \rightarrow 0$ was calculated in 21-25]; it turns out here that the numbers $\lambda$ satisfying (1) fill a half-strip in the complex plane entirely, while the actual spectrum is discrete and concentrates near some graph. The results of these papers can be reformulated in terms of the quantization rules on Riemann surfaces as follows. Consider a Riemann surface $\Lambda$ in the complex phase space $\Phi=(\mathbb{C} / 2 \pi \mathbb{Z}) \times \mathbb{C}$ with coordinates $(x, p)$, where $\Lambda$ is given by the equation

$$
p^{2}+\imath V(x)=\lambda
$$

this surface is obtained by gluing together two cylinders of the variable $x$ along finitely many cuts, namely, the zeros of the trigonometric polynomial $V$ are joined to one another and to the points at infinity. The results of the papers mentioned above imply the following assertion.

Theorem 2. The spectrum of the Schrödinger operator concentrates in the $O\left(h^{2}\right)$-neighborhood of the set given by the family of equations

$$
\frac{1}{2 \pi h} \int_{\gamma} p d x=m+\frac{\mu}{4}
$$

where $\gamma$ is some cycle on surface $\Lambda, \mu \in\{0,2\}$, and $m=O(1 / h)$ is an integer.

Remark 5. In contrast to the self-adjoint case, the quantization condition must hold on only one cycle in the given family of cycles, and different cycles determine different parts of the spectrum.

Remark 6 . Separating the real and imaginary parts in equations (5) we obtain the system

$$
\begin{aligned}
\Im \int_{\gamma} p d x & =0, \\
\Re \frac{1}{2 \pi h} \int_{\gamma} p d x & =m+\frac{\mu}{4} .
\end{aligned}
$$

The first equation does not depend on $h$. The combination of these equations for different cycles defines a set of analytical curves in the complex plane $\lambda$, the so-called spectral graph. The second equation defines a discrete set of asymptotic eigenvalues; for a fixed cycle $\gamma$, these eigenvalues are concentrated near the corresponding edge of the spectral graph.

Remark 7. In [21-25], examples of spectral graphs for specific surfaces $\Lambda$ are presented. In particular, if $V(x)=\cos x$, then surface $\Lambda$ is homeomorphic to a torus with two punctures; the corresponding spectral graph consists of three edges corresponding to the three cycles in the surface and has the shape shown in Fig. 1. If

$$
V=\cos x+\cos 2 x,
$$

then the surface is homeomorphic to a pretzel with two punctures (a sphere with two handles and with two disks removed); the corresponding spectral graph is shown in Fig. 2 and consists of five edges (note that the one-dimensional homology of $\Lambda$ is the fivedimensional in this case).

Remark 8. The equations for the asymptotic eigenvalues can be represented by explicit formulas

$$
\int_{x_{j}}^{x_{k}} \sqrt{\lambda-\imath V(x)} d x=\pi h\left(m_{k j}+\mu / 4\right)
$$

where $m_{k j}$ are integers, $\mu \in\{0,2\}$, and $x_{k}$ and $x_{j}$ are zeros of the integrand. In this case, the equation

$$
\Im \int_{x_{j}}^{x_{k}} \sqrt{\lambda-\imath V(x)} d x=0
$$




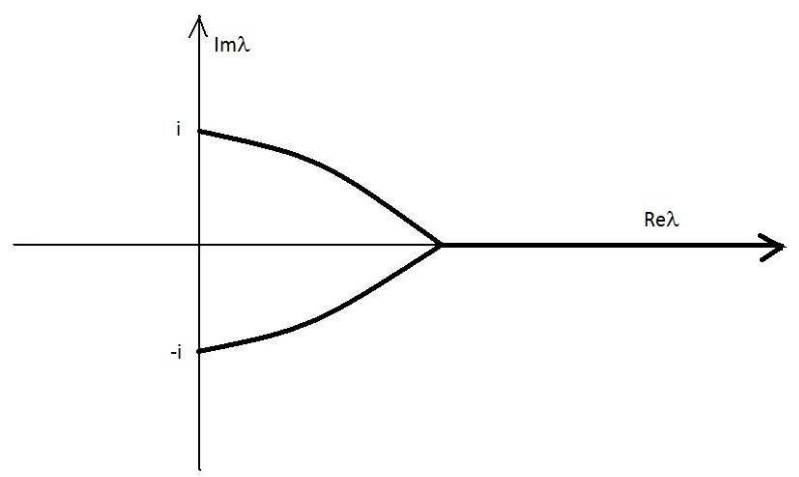

Figure 1. Spectral graph for the case $V=\cos x$

defines the edges of the spectral graph, and the spectral points are defined by the equations:

$$
\Re \int_{x_{j}}^{x_{i}} \sqrt{\lambda-\imath V(x)} d x=\pi h\left(m_{i j}+\mu / 4\right) .
$$

Remark 9. Integer $\mu$ is the analog of the Maslov index; however, the definition of this number is quite different. Namely, $\mu(\gamma)$ equals the index of intersection of the cycle $\gamma$ with the pull-back of the real circle $\Im x=0$ with respect to the projection $(x, p) \rightarrow x$.

\section{Equation OF MAGNETIC INDUCTION}

The spectral problem for the operator of induction,

$$
\begin{gathered}
h^{2} \triangle B-\{v, B\}=-\lambda B, \\
\operatorname{div} B=0
\end{gathered}
$$

arises when describing the magnetic field in a conductive liquid (in particular, the magnetic fields of planets, stars, and galaxies, see, e.g., 9]). Here, $v$ stands for a given smooth divergence-free field on a Riemannian manifold $M, \Delta$ for the Laplace-Beltrami operator, and $B$ is the desired vector field (the magnetic field). Parameter $h$ characterizes the resistance in the liquid, and the passage to the limit as $h \rightarrow 0$ corresponds to high conductivity.

Clearly, the spectrum of the operator of induction depends substantially on the manifold $M$ and on the field $V$ and can be computed efficiently in special situations only. Below we consider a special case of this kind, namely, a two-dimensional surface of revolution with the flow along the parallels. This case was discussed in detail in [24] (see also [26, 27]); we present the main results only. Recall that a two-dimensional compact surface of revolution is diffeomorphic either to a torus or to a sphere.

\subsection{Torus}

The torus is obtained by rotating a smooth closed curve around an axis that does not intersect the curve, and the metric is of the form

$$
d s^{2}=d z^{2}+u^{2}(z) d \varphi^{2},
$$

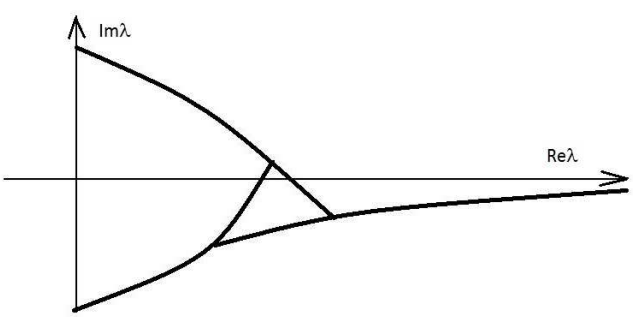

FiguRE 2. Spectral graph for the case $V=\cos x+$ $\cos 2 x$

where $z$ stands for the arc length parameter on the rotating curve, $u(z)$ for the distance of the point to the axis of rotation (we assume that $u$ is a trigonometric polynomial), and $\varphi$ for the angle of rotation. We assume that field $v$ is directed along the parallels, $v=a(z) \frac{\partial}{\partial \varphi}$, where $a$ is a trigonometric polynomial, in which case, the variables in the spectral equation can be separated and the asymptotic behavior of the spectrum can be calculated by using equations similar to (5). The Riemann surface $\Lambda$ is given by the equation

$$
p^{2}+i n a(z)=\lambda
$$

( $n$ is an integer constant entering the separation of variables), and the spectral graph is defined from equations (9), in which $V=n a$.

\subsection{SPHERE}

The sphere is obtained by rotating a smooth curve (the graph of a function $f(z)$ ) around the $z$ axis which intersects the curve at two points at which the tangent to the curve is perpendicular to the axis of rotation (the poles of the surface). We assume that

$$
f(z)=\sqrt{\left(z-z_{1}\right)\left(z-z_{2}\right)} k(z),
$$

where $z_{1}$ and $z_{2}$ are the poles of the surface, $k(z)$ is a polynomial, and $k(z)>0$ for $z \in\left[z_{1}, z_{2}\right]$. As far as field $v$ is concerned, it is assumed that

$$
v=a(z) \frac{\partial}{\partial \varphi}
$$

where $a(z)$ is a polynomial. The Riemann surface is given in $\mathbb{C}^{2}$ by the equation

$$
p^{2} f(z)^{2}+i n a(z)=\lambda
$$

it is punctured not only at the points at infinity but also at the zeros of $f$ (i.e., at the the poles of $M$ ). The asymptotics of the spectrum is still defined by equation (5); analytical equations (8) are replaced by the equations

$$
\int_{z_{j}}^{z_{k}} \sqrt{\left(f_{z}^{2}+1\right)(\operatorname{ina}(z)+\lambda)} d z=\pi h\left(m_{i j}+\mu / 4\right),
$$

where $z_{i}$ and $z_{j}$ are the zeros and poles of the integrand (in particular, the poles of the surface of revolution $M$ can be taken as the limits of integration). 


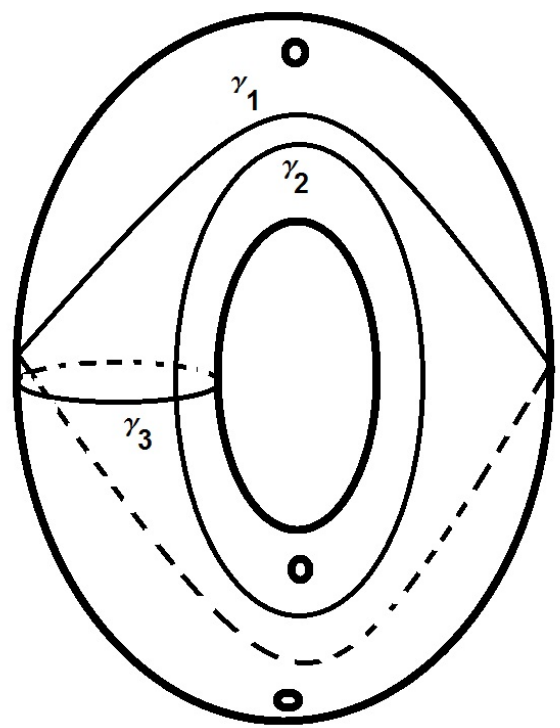

Figure 3. Cycles on the Riemann surface

As an example, consider the simplest case of the standard sphere $\left(f=\sqrt{1-z^{2}}\right)$ and take $a(z)=z$. In this case, the Riemann surface is homeomorphic to the torus with three punctures, namely, at the points $z= \pm 1$ and at the point at infinity. The cycles are depicted in Fig. 3 .

Cycle $\gamma_{1}$ goes around the points -1 and 1 , the cycles $\gamma_{2}$ and $\gamma_{3}$ go around the points $i \lambda / n,-1$ and the points $i \lambda / n, 1$, respectively. Every cycle defines the corresponding quantization conditions, which are of the form

$$
\frac{1}{\pi h} \int_{-1}^{1} \sqrt{\frac{i n z-\lambda}{1-z^{2}}} d z=\frac{1}{2}+m_{1}
$$

for cycle $\gamma_{1}$,

$$
\frac{1}{\pi h} \int_{-1}^{i \lambda / n} \sqrt{\frac{i n z-\lambda}{1-z^{2}}} d z=m_{2}
$$

for cycle $\gamma_{2}$, and

$$
\frac{1}{\pi h} \int_{1}^{i \lambda / n} \sqrt{\frac{i n z-\lambda}{1-z^{2}}} d z=m_{3}
$$

for cycle $\gamma_{3}$.

To every quantization condition, there corresponds its own sequence of eigenvalues.

Remark 10. In contrast to the preceding section, the quantization conditions corresponding to a surface of revolution involve an integer $n$ (the constant arising in the course of the separation of variables). The asymptotic eigenvalues and the edges of the spectral graph depend on $n$; thus, the graph now consists of countably many edges. For the standard sphere and for $a=z$, this graph is shown in Fig. 4

\section{Conclusions}

We have studied the asymptotic behavior of the eigenvalues of the Schrödinger operator with complex periodic potential and of the induction operator on the

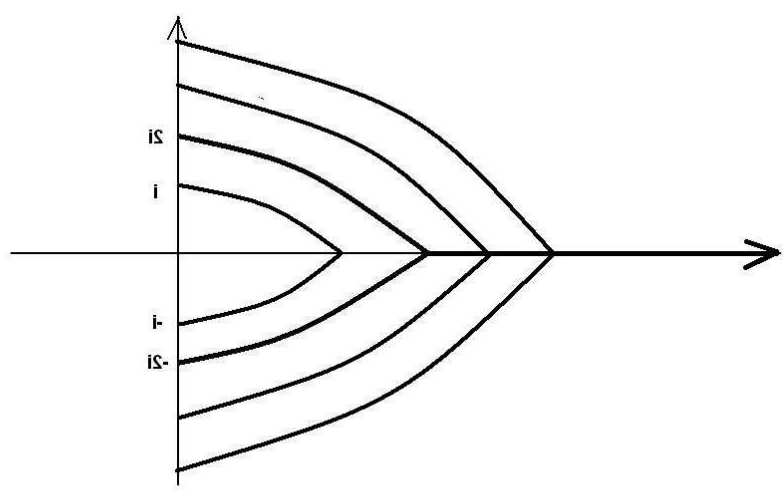

FiguRE 4. Spectral graph with countably many edges

surface of revolution. Both appear in concrete physical problems (a study of the stability of a viscous fluid, a description of the magnetic fields in stars and galaxies, statistical mechanics of Coulomb gas etc.) We show that semiclassical asymptotics of the spectrum can be computed with help of quantization conditions on the corresponding Riemann surface. We discuss the relation of these equation to the standard EBK - Maslov quantization: equations should be considered for different cycles of the surface separately and the Maslov index should be replaced by the index of intersection with the pull-back of the real circle.

\section{ACKNowledGements}

Our research was supported by a Grant from the Government of the Russian Federation for state support for scientific research carried out under the supervision of leading scientists at the Lomonosov Moscow State University Federal Budget Educational Institution of Higher Professional Education, according to Agreement 11.G34.31.0054, and also by the Russian Foundation for Basic Research under Grants 09-01-12063-ofi-m, 11-01-00937-a, and 13-01-00664, by the Program in support of leasing scientific schools (under Grant 3224.2010.1), and a grant "My First Grant" project 12-01-31235. The authors thank the referees for very useful recommendations.

\section{REFERENCES}

[1] V. P. Maslov. Asymptotic Methods and Perturbation Theory. MGU, 1965.

[2] V. P. Maslov, M. V. Fedoryuk. Quasiclassical Approximation for the Equations of Quantum Mechanics. Nauka, 1976.

[3] E. B. Davies. Pseudospectra of differential operators. Operator Theory 43:243-262, 2000.

[4] M. A. Evgrafov, M. V. Fedorjuk. Asymptotic behavior of solutions of the equation $w^{\prime \prime}-p(z, \lambda) w=0$ as $\lambda \rightarrow \infty$ in the complex $z$-plane. Uspekhi Mat Nauk 21(2):3-50, 1966 .

[5] M. V. Fedoryuk. Asymptotic Analysis: Linear Ordinary Differential Equations. Springer-Verlag, 1993.

[6] I. T. Gohberg, M. G. Krein. Introduction to the Theory of Linear Nonself-adjoint Operators. American Mathematical Society, 1969. 
[7] L. N. Trefethen. Pseudospectra of linear operators. ISIAM 95: Proceedings of the Third Int Congress of Industrial and Applied Math pp. 401-434, 1996.

[8] R. G. Drazin, W. H. Reid. Hydrodynamic Stability. Cambridge, 1981.

[9] Y. B. Zel'dovich, A. A. Ruzmaikin. The hydromagnetic dynamo as the source of planetary, solar, and galactic magnetism. Uspekhi Fiz Nauk 152(2):263-284, 1987.

[10] C. M. Bender, B. K. M. Dorje C. Brody, Hugh F. Jones. Faster than Hermitian quantum mechanics. Phys Rev Lett 98, 2007. DOI: 10.1103/PhysRevLett.98.040403

[11] T. Gulden, A. K. Michael Janas, Peter Koroteev. Statistical mechanics of Coulomb gases as quantum theory on Riemann surfaces. JETP 144(9), 2013. DOI: $10.7868 / \mathrm{S} 0044451013090125$

[12] S.-A. Stepin. Nonself-adjoint singular perturbations: a model of the passage from a discrete spectrum to a continuous spectrum. Russ Math Surv 50(6):1311-1313, 1995.

[13] A. A. Shkalikov. On the limit behavior of the spectrum for large values of the parameter of a model problem. Math Notes 62(5):796-799, 1997. DOI: 10.4213/mzm1688

[14] A. A. Arzhanov, S. A. Stepin. Semiclassical spectral asymptotics and the Stokes phenomenon for the Weber equation. Dokl Akad Nauk 378(1):18-21, 2001.

[15] A. A. Shkalikov, S. N. Tumanov. On the limit behaviour of the spectrum of a model problem for the Orr-Sommerfeld equation with Poiseuille profile. Izv Math 66(4):829-856, 2002. DOI: 10.4213/im399

[16] A. V. D'yachenko, A. A. Shkalikov. On a model problem for the Orr-Sommerfeld equation with linear profile. Funktsional Anal i Prilozhen 36(3):228-232, 2002. DOI: $10.4213 /$ faa208

[17] A. A. Shkalikov. Spectral portraits of the Orr-Sommerfeld operator with large reynolds numbers. J Math Sci 124(6):5417-5441, 2004. DOI: 10.1023/B:JOTH.0000047362.09147.c7

[18] S. A. Stepin, V. A. Titov. On the concentration of spectrum in the model problem of singular perturbation theory. Dokl Math 75(2):197-200, 2007.
[19] V. I. Pokotilo, A. A. Shkalikov. Semiclassical approximation for a nonself-adjoint Sturm-Liouville problem with a parabolic potential. Math Notes 86(3):442-446, 2009. DOI: $10.4213 / \mathrm{mzm} 8506$

[20] L. K. Kusainova, A. Z. Monashova, A. A. Shkalikov. Asymptotics of the eigenvalues of the second-order nonself-adjoint differential operator on the axis. Mat Zametki 93(4):630-633, 2013. DOI: $10.4213 / \mathrm{mzm} 10173$

[21] S. V. Galtsev, A. I. Shafarevich. Spectrum and pseudospectrum of nonself-adjoint Schrödinger operators with periodic coefficients. Mat Zametki 80(3):456-466, 2006. DOI: $10.4213 / \mathrm{mzm} 2821$

[22] S. V. Galtsev, A. I. Shafarevich. Quantized Riemann surfaces and semiclassical spectral series for a nonself-adjoint Schrödinger operator with periodic coefficients. Theor Math Phys 148(2):206-226, 2006. DOI: $10.4213 /$ tmf2081

[23] A. I. Esina, A. I. Shafarevich. Quantization conditions on Riemannian surfaces and the semiclassical spectrum of the Schrödinger operator with complex potential. Mat Zametki 88(2):209-227, 2010. DOI: $10.4213 / \mathrm{mzm} 8803$

[24] A. I. Esina, A. I. Shafarevich. Asymptotics of the spectrum and the eigenfunctions of the operator of magnetic induction on a two-dimensional compact surface of revolution. Mat Zametki (in print) 2013. DOI: $10.4213 / \mathrm{mzm} 10424$

[25] A. I. Esina, A. I. Shafarevich. Analogs of Bohr-Sommerfeld-Maslov quantization conditions on Riemann surfaces and spectral series of nonself-adjoint operators. Russian Journal of Mathematical Physics 20(2):172-181, 2013. DOI: 10.1134/S1061920813020052

[26] H.Roohian, A. Shafarevich. Semiclassical asymptotics of the spectrum of a nonself-adjoint operator on the sphere. Russ J Math Phys 16(2):309-315, 2009. DOI: 10.1134/S1061920809020150

[27] H. Roohian, A. I. Shafarevich. Semiclassical asymptotic behavior of the spectrum of a nonself-adjoint elliptic operator on a two-dimensional surface of revolution. Russ J Math Phys 17(3):328-334, 2010. DOI: $10.1134 / \mathrm{S} 1061920810030064$ 